\title{
EL TOYOTISMO COMO SISTEMA DE FLEXIBILIZACIÓN DE LA FUERZA DE TRABAJO. UNA MIRADA DESDE LA CONSTRUCCIÓN DE PRODUCTIVIDAD EN LOS SUJETOS TRABAJADORES DE LA FÁBRICA JAPONESA $(1994-2005)^{1}$
}

The toyotism as flexibilization system of the working force. A look from the productivity construction of subject workers at the Japanese factory (1994-2005)

Diego Álvarez Newman ${ }^{2}$

Universidad de Buenos Aires, Buenos Aires, Argentina.

Recibido: 28 de marzo de 2012. Aprobado: 25 de octubre de 2012.

\section{RESUMEN}

La concepción toyotista ha sido estudiada por diversos autores desde una perspectiva crítica remarcando la flexibilización laboral en cada una de sus dimensiones. Nuestro enfoque dialogará con aquellos que hacen hincapié en el sistema de producción que se pone en marcha en el proceso de trabajo, especificando en las formas de construcción de sujetos trabajadores. El Sistema Toyota de producción opera sobre la fuerza de trabajo mediante dos formas que se complementan. Por un lado se encuentran las bases objetivas de la productividad del sujeto, en donde el cuerpo y el tiempo de trabajo son sometidos mediante dispositivos de control. Por el otro se incentiva a la productividad mediante dispositivos tendientes al involucramiento de los sujetos en los objetivos de

$1 \quad$ En este artículo se presentan avances que se inscriben en investigaciones que venimos llevando adelante en el marco del proyecto UBACyT "Estrategias de disciplinamiento laboral y resistencia en el trabajo: disputas político-cultural dentro y fuera de la fábrica", con sede en la Facultad de Ciencias Sociales, Universidad de Buenos Aires; y del proyecto PIP "Prácticas hegemónicas corporativas y disciplinamiento laboral: saberes, prácticas y posiciones de los trabajadores en contextos de racionalización y fragmentación en el trabajo", con sede en el CEIL CONICET. En el presente artículo se realiza un análisis de los mecanismos utilizados por el toyotismo para lograr una flexibilización de la fuerza de trabajo que conduzca a un mayor aumento de la productividad empresarial. Para ello se retoman elementos desarrollados en Álvarez (2012), enfocando tales aspectos desde una óptica flexibilizadora.

2 Licenciado en Sociología, becario doctoral de la Facultad de Ciencias Sociales de la Universidad de Buenos Aires. Correo electrónico: diegonewman@hotmail.com. 
productividad y calidad de la empresa. Estas dos formas de construcción de "sujetos productivos" se desarrollan en simultáneo y dan como resultado la "eficacia" del toyotismo como modelo de producción. Se tomarán como fuentes de datos fundamentalmente los Convenios Colectivos de Trabajo firmados por la empresa Toyota Argentina Sociedad Anónima y el Sindicato de Mecánicos y Afines del Transporte Automotor entre el período 1994-2005. Se recurrirá en casos específicos a documentos internos, tanto de la fábrica como del sindicato.

PALABRAS CLAVE: Toyotismo, productividad, flexibilización laboral.

\begin{abstract}
The toyotism conception of the productivity has been study by different authors from a critic perspective pointing the working flexibilization in each one of its dimensions. Our look will speak to those who make a point at the production system that starts the working process, specifying in the forms of construction of workers subjects within the Japanese factory at the Argentinean location. The Toyota Production System works over the working labor by two complementary forms. On one side we have the objectives bases of the productivity subject from where the working body and time are measure under control mechanism that interact in a straight way with the workers. On the other side, they encourage productivity thru mechanism that involves the productivity objectives and quality of the company. These two forms of construction of "productive subjects" are developed simultaneously and have as a result the "efficiency" from the toyotism as a production model. It will be taken as data source specially the Working Agreement sign between the Toyota Company in Argentina and the Union of mechanics of Auto Transport. Also, we'll incur in specific cases into internal documents from the factory as from the Union.
\end{abstract}

KEYWORDS: Toyotism, productivity, labor flexibility.

\title{
I. INTRODUCCIÓN
}

La concepción toyotista de la producción ha sido estudiada por diversos autores desde una perspectiva crítica, ya sea haciendo hincapié en su sistema de producción (Juárez 2002; Coriat 1992), en las transformaciones en el trabajo (Gorz 1998), en las transformaciones de las relaciones laborales (Battistini 2001), en la construcción de identidades 
laborales y subjetividades (Battistini 2005; Escobar 2004; Wortman 2003) y en la comparación de modelos productivos (Novick, Yoguel, Catalano y Albornoz 2004; Lipietz 1994). Desde la teoría marxista pueden encontrarse textos que describen el toyotismo para analizar las metamorfosis en el mundo del trabajo (Antunes 2003), que focalizan en las formas de explotación que se ponen en juego en el proceso de producción (Martínez 1997; Watanabe 1997), que describen la innovación tecnológica en la fábrica en el marco de la lucha de clases (Ichiyo 1997) y que observan las formas de reestructuración productiva y la cuestión sindical (Alves 1998; Totsuka 1997).

Si bien en todos estos enfoques se remarca la flexibilización laboral en las distintas dimensiones del toyotismo, nuestra perspectiva dialogará con las que ponen el foco en el sistema de producción que se pone en marcha en el proceso de trabajo. Es decir, en el presente artículo se abordará la flexibilización desde las complejas modalidades de construcción de productividad en los sujetos trabajadores ${ }^{3}$ en la fábrica japonesa. La hipótesis que se sostiene es que la eficiencia del Sistema Toyota de Producción se logra mediante la implementación de sofisticados dispositivos que forman maneras de hacer y de ser flexibles y tendientes a un aumento continuo de la productividad en los sujetos trabajadores de la planta.

¿Cómo se construye el sujeto trabajador toyotista? ¿Cuáles son sus bases objetivas y los aspectos cualitativos de su productividad? ¿Por qué es una forma flexible de construcción de productividad? ¿Qué rol cumple el sindicalismo en este proceso de flexibilización de la fuerza de trabajo?

El Sistema Toyota de Producción opera sobre la fuerza de trabajo mediante dos formas que se complementan. Por un lado se encuentran las bases objetivas de la productividad del sujeto, en donde el cuerpo y el tiempo de trabajo son sometidos mediante dispositivos de control. Por el otro, se incentiva a la productividad mediante dispositivos tendientes al involucramiento de los sujetos en los objetivos de productividad y calidad de la empresa. Estas dos formas de construcción de "sujetos productivos" se desarrollan en simultáneo y dan como resultado la "eficacia" del toyotismo como modelo de producción.

3 La noción de sujetos trabajadores se entiende en relación al concepto de disciplina elaborado por Michel Foucault (2002:141). En este texto el autor aborda a la disciplina como forma de sujeción que toma a los cuerpos como objeto y blanco de poder. Es decir, como un control minucioso de las operaciones corporales que garantizan la sujeción constante de sus fuerzas y le imponen una relación de docilidad-utilidad. "Un "arte" del cuerpo humano que no tiende únicamente al aumento de sus habilidades ni tampoco a hacer más pesada la sujeción, sino a la formación de un vínculo que lo hace tanto más obediente cuanto más útil y al revés." En este sentido, la modalidad toyotista de sujeción implica aumentar la capacidad productiva del sujeto trabajador maximizando la voluntad de obediencia a los objetivos de la empresa mediante sofisticados dispositivos basados en la filosofía gerencial de la mejora continua de la calidad. 
Como estrategia de análisis se comenzará por reseñar brevemente el Sistema Toyota de Producción, para luego describir y analizar las bases objetivas de la construcción de los sujetos trabajadores y el involucramiento como factor de productividad. Por último, se analizará el papel desempeñado por el Sindicato de Mecánicos y Afines del Transporte Automotor (SMATA) frente a este sistema flexible de producción.

En cuanto al abordaje metodológico, se tomarán como fuentes de datos fundamentalmente los Convenios Colectivos de Trabajo firmados por la empresa Toyota Argentina Sociedad Anónima (TASA) y el SMATA entre el período 1994-2005. Asimismo, se recurrirá en casos específicos a documentos internos, tanto de la fábrica como del sindicato.

\section{LOS PRINCIPIOS BÁSICOS DEL SISTEMA TOYOTA DE PRODUCCIÓN}

El Sistema Toyota de Producción (STP), en tanto tecnología de gestión del trabajo, es un sistema complejo de racionalización que interviene mediante sofisticados dispositivos sobre el espacio fabril y su organización técnica, sobre los trabajadores y sobre las prácticas sindicales (Álvarez 2012:46).

La construcción de la productividad toyotista tiene que ver tanto con formas "eficientes" de organizar el espacio como con un control del tiempo y de los movimientos de los trabajadores en la línea de producción. Al igual que en el taylorismo-fordismo, el sistema está basado en la eliminación de tiempos y acciones improductivas (desperdicio) para lograr la más alta productividad.

Sin embargo, lo que da sentido al toyotismo por dentro de los espacios de trabajo son menos las rigideces que las formas flexibles de producción. Son los dispositivos de control que operan en el STP los que vehiculizan los principios fundamentales sobre los que se asienta la filosofía Toyota. Estos principios tienen que ver con la orientación al mercado de la producción, con el alineamiento de los trabajadores a los objetivos de la empresa y con la voluntad de mejorar continuamente la productividad y la calidad del producto.

Es el just in time, el dispositivo que permite una adaptación total tanto técnica como social a los vaivenes del mercado. Esto es, producir solo lo necesario en el momento justo y en la cantidad necesaria ${ }^{4}$ para que no haya desperdicio de tiempo ni de piezas.

4 Toyota Argentina Sociedad Anónima, documentos "Acerca de Toyota" y "Conociendo a Toyota, Sistema de Producción Toyota". 
Ineludiblemente el sistema debe ser flexible para poder lograr una mejor adaptabilidad, tanto en términos de productividad como de calidad del producto. La constante apelación a la calidad que se hace desde el STP implica producir para que cada cliente compre el vehículo de la especificación y color que desea y lo obtenga en el plazo más breve posible. ${ }^{5}$ Pero productividad y calidad no deben entenderse de manera disociada sino que son parte de un mismo proceso de adaptación al mercado, tanto en términos objetivos, en el sentido de gasto de fuerza de trabajo y tiempo, como en términos cualitativos, en el sentido de subjetividades que deben formarse en un saber ser y saber hacer frente a los requerimientos de la demanda.

A su vez, el jidoka es el dispositivo técnico que vehiculiza el funcionamiento del just in time, ya que permite la eliminación de defectos y desperdicios en tiempo real mediante la preparación de una línea de producción con capacidad para detenerse cuando se detectan problemas, tales como el mal funcionamiento de los equipos, retraso en el trabajo o problemas de calidad.

La adaptación de los trabajadores a las formas flexibles de producción se moviliza mediante el involucramiento en los objetivos de la empresa. Se ponen en juego variadas técnicas de normalización (que serán especificadas más adelante), que se distinguen de los dispositivos electrónicos de control técnico sobre las líneas de producción en que no buscan solo un sometimiento directo de la fuerza de trabajo a partir de la imposición de ritmos y movimientos, sino que intervienen sobre la construcción de valores que serán de suma utilidad para incrementar las habilidades de los trabajadores y así lograr una mayor productividad. La doctrina de la mejora continua de la productividad, la calidad total y la satisfacción del cliente también se ponen en práctica mediante dispositivos de formación continua que alientan a los trabajadores a involucrarse con la productividad y la calidad.

El toyotismo es mucho más que una forma de organizar el proceso de trabajo en términos técnicos, ya que supone una manera de relación entre capital y trabajo en donde lo ideológico-cultural adopta formas totalmente distintas al taylorismo-fordismo. En términos políticos-ideológicos, desde el toyotismo no se reconoce que la relación entre capital y trabajo sea contradictoria de por sí. Más bien, la lógica toyotista reconoce la posible existencia de conflictos, pero estos no tienen que ver con antagonismos de clase. El toyotismo pregona la construcción de una cultura de trabajo que sustituya estos antagonismos por los "consensos" entre "colaboradores".

5 Toyota Argentina Sociedad Anónima, documentos "Acerca de Toyota" y "Conociendo a Toyota, Sistema de Producción Toyota". 
De esta manera, la construcción de la productividad toyotista tiene que ver con una conjunción entre el típico uso intensivo de la fuerza de trabajo en términos de controles de ritmos y movimientos, y la movilización de dispositivos que intervienen sobre cuestiones "ideológicas" y subjetivas que pretenden lograr el efecto de involucramiento en la producción.

\section{LAS BASES OBJETIVAS DE LA CONSTRUCCIÓN DE PRODUCTIVIDAD EN LOS SUJETOS TRABAJADORES}

\section{La fábrica mínima}

El uso flexible de los trabajadores constituye uno de los pilares fundamentales del STP, ya que es lo que permite una mejor adaptabilidad de la organización para "producir con los efectivos necesarios y en el momento preciso", y así poder responder a las variaciones del mercado.

Pero no es posible comprender la flexibilización del sistema sin el concepto de "fábrica mínima". El sistema, además de estructurarse a partir de un número mínimo de trabajadores que se puede ir ampliando mediante horas extras o nuevas contrataciones, siempre de acuerdo a las variaciones del mercado, se estructura también sobre una fuerte desconcentración productiva y subcontratación.

En sus análisis sobre la cuestión de la subcontratación en el toyotismo, Benjamín Coriat (1992:104) sostiene que en la casa matriz japonesa solo el 26,5 por ciento de los componentes se fabrican internamente, mientras que el 73,5 por ciento restante lo proveen empresas subcontratadas. En valores absolutos, en 1986 la casa matriz de Toyota contaba con más de 36.000 empresas subcontratistas. ${ }^{6}$

Pero la tercerización no solo opera sobre las empresas fabricantes de componentes para la producción, también lo hace sobre los trabajadores y los puestos de trabajo dentro de la fábrica. Diversas actividades, fundamentalmente las que están ligadas a los servicios, también están tercerizadas, estando su personal excluido del Convenio Colectivo de Trabajo.

6 Ricardo Antunes (2005:39) sostiene que el porcentaje de subcontratación en la "empresa fordista" era prácticamente el inverso: el 75 por ciento de la producción era realizada internamente. Este dato muestra claramente el cambio de paradigma productivo que significó el toyotismo. 
Estarán excluidos los empleados de empresas proveedoras especializadas, que no correspondan a las actividades normales y específicas de TOYOTA ARGENTINA, a saber: construcción, reparación, modificación y mantenimiento de obras civiles; sistemas de vigilancia y seguridad; servicio de limpieza y mantenimiento provisto por terceros; preparación, distribución y servicio de comidas; manejo, recepción y despacho de cargas y materiales; servicios médicos y enfermería; servicio de informática y de transporte (Convenio Colectivo de Trabajo $n^{\circ}$ 190/96).

Este estilo de desconcentración productiva y tercerización responde a la manera de organizar el trabajo del STP a partir de un número mínimo de operarios polivalentes que sí están afectados a las "actividades normales" de la empresa. El concepto de "fábrica mínima" opera como flexibilización en dos sentidos: a la vez que elimina, desconcentra y terceriza actividades, incluso las que se desarrollan dentro de la fábrica, sobrecarga de tareas a los trabajadores comprendidos en el Convenio Colectivo. Para la efectiva aplicación del STP la flexibilización implica necesariamente la polivalencia como categoría que articula y refuerza el logro de una mayor productividad en el marco de la "fábrica mínima".

\section{La polivalencia}

La polivalencia funcional es la categoría que permite el uso flexible de la fuerza de trabajo dentro de la fábrica, ya que interviene como herramienta para poder utilizar a los trabajadores en diversos puestos de trabajo. En los convenios colectivos de trabajo, al ser la maximización de la productividad uno de los patrones más importantes que guían al STP, el poder de TASA de distribuir tareas de acuerdo a las necesidades operativas de la empresa es total. Solo existen dos categorías de trabajadores para llevar adelante el proceso productivo para un amplio universo de tareas.

\section{Operario polivalente o team member}

Operarios con conocimiento de técnicas del Sistema de Producción Toyota. Podrá desempeñarse indistintamente en cualquiera de las siguientes funciones, como ser: ensamble de vehículos (comprendiendo todas y cualquiera de las operaciones para tal fin), pintura, soldadura, tapicería, estampado, montaje y armado de conjuntos y subconjuntos y equipamientos de vehículos, mantenimiento de herramientas, maquinarias industriales y robótica, control de procesos productivos y de calidad de procesos, partes y vehículos, preparación de herramientas y maquinarias para el trabajo, manejo de materiales, orden 
y limpieza de lugares de trabajo y toda tarea relacionada específicamente con la fabricación de vehículos.

\section{Operario polivalente líder de célula o team leader}

Será aquel operario que deberá conocer, desempeñar y/o asistir en cualquiera de las funciones antes mencionadas. También deberá ser apto para capacitar a los operarios de la categoría precedente en todas y cada una de las funciones y tareas de la célula. Coordinará los equipos de trabajo en donde se desempeñe. Asimismo reemplazará en todos los casos que sea necesario y cuando las necesidades operativas y de producción así lo requieran, a los operarios polivalentes en ausencia de estos (Dirección del Trabajo 1996).

Este achatamiento de las categorías permite contar con trabajadores polivalentes y multioficios para el adecuado funcionamiento flexible del sistema, en términos de lograr una mayor productividad de la fuerza de trabajo. La diferencia que se encuentra entre la categoría operario polivalente y la de operario polivalente líder radica en que el segundo, además de conocer todas las funciones del proceso productivo, posee la habilidad de $c a-$ pacitar y coordinar a las células de trabajo. En este sentido, la realización de tareas de formación continua adquiere una gran importancia en el funcionamiento del STP, ya que el uso intensivo de la fuerza de trabajo en términos objetivos se ve reforzado por una matriz pedagógica tendiente a incentivar la productividad del sujeto trabajador. Así, se pretende calibrar de manera "eficaz" tanto la adaptación corporal como mental de los trabajadores a los ritmos del proceso de trabajo.

Si bien en el Convenio Colectivo de Trabajo firmado entre las partes en el año 2001 se establecen seis niveles para la categoría operario polivalente y cuatro para la de operario polivalente líder, estas no afectan a la polifuncionalidad de las tareas de producción, sino a las remuneraciones y a la antigüedad (Dirección del Trabajo 2001a).

Las tareas, funciones y categorías incluidas en el presente convenio se las considera polivalentes, de modo que el trabajador deberá realizar toda tarea, función o actividad que se le asigne - de conformidad con las pautas de la legislación vigente-, acorde a su capacitación y teniendo en cuenta para ello "el mapa de habilidades", que forma parte del TPS (Dirección del Trabajo 2001b).

Tanto en el mencionado convenio de 2001 como en el que firmaron las partes en 2005, encontramos una cierta revalorización de la antigüedad con respecto al convenio firmado en 1996. Sin embargo, el traspaso de un nivel de categoría a otro no se produce de manera auto- 
mática por antigüedad, sino que se encuentra sometido a sofisticados criterios de evaluación permanente por parte de la empresa. La antigüedad representa solo uno de los siete criterios de evaluación para el traspaso de nivel, mientras que los seis restantes hacen referencia a objetivos de calidad y productividad (Dirección del Trabajo 2005a). La introducción de estos niveles a las dos categorías polivalentes mencionadas refuerza el involucramiento en la producción por vía de las remuneraciones adicionales por productividad y calidad.

El haber agregado niveles a las dos categorías de operarios polivalentes, además de no tener influencia sobre la multiplicidad de funciones que exige la empresa a los trabajadores, no impacta directamente sobre la antigüedad y las remuneraciones porque queda bajo el control de la dirección de la empresas a partir de evaluaciones de desempeño y cupo (Dirección del Trabajo 2005b).

\section{La jornada de trabajo}

La jornada de trabajo es tal vez un eje fundamental de la flexibilización toyotista, ya que marca el tiempo que los trabajadores se ven afectados a las tareas de producción. Si con la polivalencia la empresa puede disponer de los trabajadores para rotarlos por diferentes puestos de trabajo, la jornada flexible permite un uso del tiempo de trabajo que se ajusta a las necesidades de productividad del momento. Está compuesta por un turno de trabajo que va de las 08:00 a las 17:10 hrs. y puede ser modificada arbitrariamente por la empresa de acuerdo a las necesidades del mercado.

$\mathrm{Al}$ personal que ingrese a TOYOTA ARGENTINA le será asignado un turno de trabajo en concordancia con los turnos establecidos en este artículo. [...] Cuando las necesidades del trabajo o razones económicas o de productividad lo requieran, la Empresa podrá disponer el trabajo por turnos rotativos y/o por equipo (Dirección del Trabajo 2005c).

La posibilidad de un mayor aprovechamiento de la jornada en términos de productividad mediante la incorporación de más turnos y su rotación se conjuga con la posibilidad que tiene la empresa de utilizar las dos pausas diarias, de 10 minutos cada una, que tienen los trabajadores para tratar temas específicos de productividad y calidad (Dirección del Trabajo 2005d).

Asimismo, la jornada de trabajo en la fábrica japonesa se encuentra anualizada, lo que permite a la empresa disponer de un uso del tiempo de trabajo aún más flexible. Al ser una jornada de 2.133 horas anuales, con un sistema de débitos y créditos de horas (Di- 
rección del Trabajo 2005e), la empresa puede "calibrar" mejor la utilización del tiempo en función de la demanda del mercado.

La complejidad del STP supone no disociar en el análisis las distintas categorías que se están poniendo en juego. Si la polivalencia permite un uso discrecional de la fuerza de trabajo en términos de la utilización por puestos, la jornada anualizada permite una mejor adaptación del tiempo de trabajo a los requerimientos del mercado. En épocas de mayor demanda la empresa puede prolongar la jornada hasta los límites diarios que fijan las leyes laborales y extenderla aún más mediante la incorporación de turnos rotativos.

Las bases objetivas de la productividad toyotista se asientan sobre la polivalencia, la flexibilización de la jornada de trabajo y la tercerización y la desconcentración productiva. Sin embargo, la sofisticada construcción de la productividad que lleva adelante el sistema pone de relieve aspectos cualitativos que se focalizan en la necesidad de involucrar a los trabajadores en las tareas de producción.

\section{EL INVOLUCRAMIENTO COMO FACTOR DE PRODUCTIVIDAD EN LOS SUJETOS TRABAJADORES}

La base objetiva de la flexibilización de la fuerza de trabajo, vía la polivalencia y el control del tiempo de trabajo, se complementa con factores que intervienen de manera indirecta sobre el cuerpo de los trabajadores.

Paralelamente al núcleo duro de la construcción de productividad en los sujetos operan factores de carácter "filosófico" referidos a la mejora continua de la calidad. En términos de la construcción de la productividad en los sujetos trabajadores, la mejora continua busca incentivar una actitud proactiva en el trabajo, es decir, fomentar mediante técnicas de formación continua el involucramiento en la producción.

En este sentido, el sometimiento directo de la fuerza de trabajo encuentra sus fundamentos de legitimidad en la filosofía de la mejora continua, ya que es tan importante para el sistema de producción una disposición flexible de los cuerpos como el logro de conductas adaptadas a los vaivenes del mercado.

La insistencia en la fábrica que aprende de sus defectos y el entrenamiento continuo en los puestos de trabajo, hace que los espacios de producción y formación se combinen y se conjuguen constantemente. La apelación que desde el toyotismo se hace al cam- 
bio cultural apunta a lograr una mayor colaboración de los trabajadores con la empresa y ubica en el centro de la escena la conquista de su subjetividad.

Precisamente, el carácter superador del toyotismo con respecto al fordismo, más allá de los procesos técnicos de automatización, y tal como sostienen Antunes (2003:21) y Coriat (1992:23), tiene que ver con la superación de las "rigideces" en la gestión de la fuerza de trabajo. Pero esta modalidad de gestión flexible para el uso de la fuerza de trabajo viene acompañada de una base filosófico-gerencial que se impone en el terreno de la construcción de productividad también en términos culturales. Si la fábrica fordista estandarizaba mediante rígidos puestos de trabajo las maneras de hacer, la flexibilización toyotista, al tiempo que desbloquea esas maneras de hacer, forma maneras de ser en el trabajo. Precisamente, la importancia técnico-política del toyotismo radica en que pone en el centro de la escena la producción de sujetos trabajadores con un perfil determinado culturalmente por la voluntad de mejorar continuamente la productividad y la calidad de los productos.

La apuesta filosófico-cultural del toyotismo no debe entenderse por fuera del proceso de trabajo. Just in time (proceso de producción por demanda) y mejora continua de la calidad (involucramiento en la calidad del producto al servicio de la satisfacción del cliente) son dos caras de la misma moneda. Ambos constituyen la base material y cultural de la productividad toyotista.

Los dispositivos de involucramiento en la producción tienen la particularidad de que no intervienen de manera directa sobre el cuerpo-tiempo de trabajo, sino que lo hacen construyendo espacios de formación para fomentar la colaboración con la empresa.

\section{El trabajo en equipo}

Las células de trabajo constituyen un dispositivo fundamental para la formación del involucramiento en la producción. Cada célula se compone de un team leader y de ocho operarios polivalentes. Dentro de cada célula de trabajo, los team leader cumplen un rol fundamental en la transmisión de los principios del STP a los miembros polivalentes, ya que, además de un conocimiento total del proceso productivo, se destacan por ser aptos para guiar, capacitar, coordinar y controlar a cada operario de la célula. Se constituyen, así, como agentes transmisores de los saberes corporativos, aptos tanto para la producción como para el control simbólico, y capaces de asimilar la estrategia corporativa y conducir a su grupo de trabajo hacia el logro de los objetivos empresariales (Figari y Hernández 2008:16). 
Este sistema de mejora constante busca eliminar la improductividad y optimizar las habilidades de los integrantes de las células. Para ello, los problemas se resuelven a través del esfuerzo del equipo. Cuando un miembro de la célula tiene un problema, se considera que es un problema de la célula y a través del esfuerzo de esta, se busca con empeño su solución. El trabajo en equipo es un componente esencial del SPT. La comunicación es fundamental para el éxito del SPT. Para formar parte de una célula de trabajo, los miembros de esta deben entender o conocer el proceso de producción en forma integral, incluyendo la utilización de los instrumentos y herramientas, técnicas y metodologías de trabajo que a tal efecto la Empresa implemente" (Dirección del Trabajo 1996b).

La cuestión comunicacional dentro de las células de trabajo se encuentra supeditada a la eliminación continua de la improductividad. Sin la polivalencia (conocimiento integral del proceso de producción), lo comunicacional no tendría razón de ser. El hecho de que los líderes de cada célula cumplan funciones específicas de transmitir a los miembros una actitud involucrada, es un aspecto comunicacional que se encuentra vinculado a la información que cada miembro de la célula debe poseer de la totalidad del proceso de trabajo. Lo que se transmite y comunica es polivalencia en el marco de la mejora continua.

Productividad (polivalente) y comunicación al nivel de las células siguen la misma lógica de los pares just in time y mejora continua al nivel del sistema en su aspecto técnico-filosófico. Mientras la mejora continua de la calidad legitima la producción por demanda, la tarea comunicacional de los líderes busca efectos de convencimiento acerca de la actitud polivalente en los trabajadores.

Además, las células de trabajo incentivan la competitividad entre los equipos de trabajo. La flexibilización de las remuneraciones (ahondaremos más adelante) y los premios por productividad y calidad grupal refuerzan la necesidad de involucramiento, ya que el esfuerzo individual repercute en los resultados del grupo.

\section{El kaizen}

Tal como se viene sosteniendo, el uso flexible de la fuerza de trabajo vía el involucramiento en la producción se asienta también en el kaizen, que significa mejora continua. Su principal objetivo es eliminar todo aquello que no agrega valor al producto y disminuir los costos de producción. Las actividades kaizen son un espacio destinado a la búsqueda de una mejor forma de hacer las cosas, enfatizando en las operaciones de trabajo 
manual, y son realizadas por todos los trabajadores de la planta. Como en los círculos de calidad, los trabajadores deben realizar al menos dos sugerencias mensuales, por ejemplo, sobre cómo reducir tiempos de espera, de inventario, eliminar sobreproducción, etc.

[...] Este sistema parte del "principio de trabajo estandarizado", lo que implica que la Empresa establece procedimientos y normas para la realización de trabajos de sus equipos y los miembros del mismo los realizan en concordancia con esas directivas, pudiendo revisar cada equipo de empleados los procedimientos y estándares de trabajo en forma continua para obtener mejoramientos en su eficiencia, calidad y condiciones de labor, determinándose así, una vez aprobado, un nuevo estándar de trabajo (Dirección del Trabajo 2005f).

Este dispositivo constituye toda una novedad con respecto al "trabajo rutinario fordista" no solo por la movilización del intelecto de los trabajadores para mejorar la calidad, sino también por la flexibilización de los estándares de trabajo. Además, esta captura de los saberes de los trabajadores, a la vez que opera de manera individual mediante un sistema de sugerencias, permite establecer un control estadístico sobre los aportes en mejora de los grupos de trabajo y establecer estándares de mejoras necesarias, en un lapso de tiempo, para establecer nuevos criterios y objetivos de productividad y calidad. Así, la estandarización de los procesos de trabajo se flexibiliza, ya que una norma es tal hasta que se introduce una mejora y vuelve a modificarse.

Claramente es la empresa la que establece los principios de trabajo estandarizado sobre los cuales los miembros de cada célula pueden sugerir mejoras de la calidad y la productividad. Este particular sistema complejiza la separación entre trabajo de concepción y trabajo de ejecución, ya que el trabajo intelectual que los operarios pueden realizar encuentra sus límites en la definición de los objetivos por parte de la dirección de la empresa. ${ }^{7}$ Los equipos de trabajo no definen ni tienen participación en la construcción de los objetivos de calidad y productividad, sino que los que lo hacen son los encargados de la gestión estratégica de la empresa.

7 En relación a la fábrica de Volvo de Uddevalla, André Gorz (1998:45) sostiene que los obreros trabajan en equipo, que cada uno conoce varios oficios, que los equipos tienen autonomía para organizarse entre sí, que los obreros pueden sentirse responsables por la calidad del montaje, pero ni la concepción del vehículo ni la decisión de producir automóviles depende de ellos. Por lo tanto, el producto final de su trabajo sigue siendo ajeno. "Siguen al servicio de objetivos que no pueden elegir y de los cuales, en la mayoría de los casos, ni siquiera tienen conocimiento". 


\section{El sistema de remuneraciones}

Es una obviedad que el salario constituye un dispositivo de control fundamental sobre los trabajadores: sin salario no habría mercado de trabajo porque no existirían "obreros libres" en condiciones de vender su fuerza de trabajo. La complejidad del sistema de remuneraciones toyotista supone que el valor de la fuerza de trabajo se establece sobre criterios de productividad y calidad de acuerdo a desempeños individuales y colectivos de los equipos de trabajo.

Este sistema (sistema de remuneraciones variable) tiene por finalidad reconocer los mayores esfuerzos de cada célula o sector, así como también el logro de los objetivos de productividad y calidad establecidos por la Empresa. Cuando se logren estos objetivos, TOYOTA ARGENTINA abonará mensualmente a sus empleados, un adicional equivalente al 13 por ciento de la remuneración básica de cada trabajador, siempre que se cumpla con el 100 por ciento del objetivo fijado. Dicho adicional estará sujeto a variabilidad en el supuesto de que el cumplimiento del objetivo fijado sea superior o inferior al 100 por ciento (Dirección del Trabajo 2005g).

El sistema de remuneraciones variable establece que al sueldo básico mensual corresponde un adicional del 13 por ciento a partir de dos criterios establecidos por la empresa: productividad y calidad en relación al cumplimiento de la totalidad de los objetivos. La fórmula determinada para medir los objetivos contempla los siguientes factores: nivel de productividad, vehículos terminados teóricos, trabajadores comprendidos en convenio, horas trabajadas y horas extras a trabajar (Dirección del Trabajo 2005h).

Por su parte, los traspasos de niveles de categoría, tanto para el operario polivalente como para el operario polivalente líder, toman en cuenta ocho criterios de evaluación de desempeño: antigüedad en la posición, habilidades, seguridad, concepto general, círculos kaizen, sistema de sugerencias, capacitación en TWI y cupo (Dirección del Trabajo 2005i). Las evaluaciones de desempeño serán fundamentales a la hora de establecer las remuneraciones a nivel individual y a nivel grupal. Por lo tanto, estos sofisticados criterios y factores que inciden en la determinación de las remuneraciones, a la vez que flexibilizan, individualizan las relaciones de trabajo.

La construcción de la productividad de la fuerza de trabajo en el toyotismo se encuentra diversificada entre el control al trabajador individual y el equipo de trabajo. Las remuneraciones variables son un eje fundamental de la flexibilización porque es el dispositivo lo que permite premiar o castigar a los trabajadores más o menos productivos y 
más o menos involucrados; mientras que los otros dispositivos intervienen directamente sobre la productividad del cuerpo a través de la jornada de trabajo, de la intensificación de los ritmos y de la formación de actitudes vinculadas a la interiorización de la mejora continua de la calidad.

\section{LA ADHESIÓN SINDICAL AL SPT}

Esta tecnología flexible de gestión del trabajo no podría implementarse sin modalidades de regulación sobre las organizaciones sindicales de los trabajadores, ya que el conflicto es concebido desde el toyotismo como factor de limitación a la productividad.

Durante el período de exploración para la realización de la inversión en la Argentina en el año 1994, Toyota llevó adelante un proceso de selección de un sindicato que se adapte a la cultura de la empresa fundamentalmente en dos aspectos: la negociación de los convenios colectivos de trabajo a nivel de la empresa y la aceptación de las particularidades del STP. Fue así como los directivos de la empresa se inclinaron por el Sindicato de Mecánicos y Afines del Transporte Automotor (SMATA) por sobre la Unión Obrera Metalúrgica (UOM) (Battistini 2001:556).

Con la elección del SMATA como sindicato representante de los trabajadores de la planta ubicada en Zárate, Provincia de Buenos Aires, se celebró el primer Convenio Colectivo de Trabajo (190/96), con la particularidad de que fue firmado con anterioridad a la puesta en marcha de la producción. En este convenio queda de manifiesto la total colaboración del SMATA con la concepción gerencial del STP.

El S.M.A.T.A., apoyará a TOYOTA ARGENTINA, en pos de alcanzar los objetivos comunes de éxito comercial y bienestar de su personal; en particular apoyando las iniciativas que promuevan la mejora constante de la calidad y la productividad, en tanto ello redundará en la mejora de las condiciones laborales del personal representado. El S.M.A.T.A. acuerda cooperar con TOYOTA ARGENTINA en la implementación de iniciativas de capacitación y entrenamiento para los empleados de TOYOTA ARGENTINA comprendidos en el presente convenio (Dirección del Trabajo 1996c).

En esta misma lógica, la estrategia de inserción de Toyota en el país responde al denominado modelo de greenfields, ${ }^{8}$ en el cual, el perfil de trabajador a reclutar responde

8 La inserción estilo "greenfields" responde a la estrategia de elegir para la inversión de capitales zonas geográficas sin tradición industrial, la firma de convenios colectivos de trabajo "flexibles" que incluyen cálculos de jornada 
a jóvenes, en su mayoría de sexo masculino, con escolaridad secundaria y sin experiencia en el sector. Para la mitad de los trabajadores reclutados al momento de la instalación de la planta de Zárate, Provincia de Buenos Aires, era su primer empleo, mientras que, el promedio de edad del sector de los operarios y team leaders era de 23 años (Novick, Yoguel, Catalano y Albornoz 2002:8). Toyota inició un proceso de formación de la primera camada de sus jóvenes operarios en Japón con el objetivo de lograr una mejor interiorización del SPT para su aplicación en Argentina.

Asimismo, la adhesión del sindicato a la matriz teórica del SPT no se limita a la geografía de la fábrica en Zárate. En términos de formación y capacitación, el SMATA considera a la "política de calidad" como un medio estratégico para el cumplimiento de sus objetivos. Entre ellos se mencionan:

...disponer de un sistema de calidad que asegure la confiabilidad y estabilidad de la metodología de trabajo, basados en el principio de la Mejora Continua, y, capacitar, motivar y desarrollar constantemente a todo el Personal a los efectos de disponer de recursos humanos comprometidos con la Calidad del Servicio. ${ }^{9}$

La mejora continua de la calidad se va constituyendo como un principio legitimador fundamental del toyotismo, ya que es a partir de él que la filosofía Toyota se extiende no solo a otras esferas de la producción sino también a otros ámbitos como, por ejemplo, la formación sindical.

Los Centros de Formación Profesional que gestiona el SMATA siguen la misma lógica de gestión de la calidad. El Centro de Formación Profesional $N^{\circ} 8$ del SMATA tiene como visión, "consolidar a la Institución como referente sectorial reconocida por la calidad de sus acciones a partir del desarrollo de estrategias formativas innovadores, la utilización de equipamiento didáctico de última generación y la pertinencia de su oferta definida en términos de adecuación a las demandas del SMyRA"10 (Servicio de Mantenimiento y Reparación de Automotores). Mejora continua de la calidad y adaptación a la demanda son los principios fundamentales de las Instituciones de Formación Profesional que gestiona el SMATA. La lógica del toyotismo se traslada también al ámbito de la formación profesional, dejando de ser este un espacio de reivindicaciones de los trabajadores en términos

anual, reducción del número de categorías y menor participación sindical. En cuanto a la selección de personal, son jóvenes de mayor nivel educativo y sin experiencia sindical.

9 Sindicato de Mecánicos y Afines del Transporte Automotor http://www.smata.com.ar. Formación y capacitación. Política de calidad.

10 SMATA, Centro de Formación Profesional Nº: "Manual de Gestión de la Calidad Educativa". 
de actualización técnica para el trabajo, pasando a ser un espacio de formación de valores vinculados a la satisfacción del cliente y a la adaptación a la producción por demanda (just in time).

\section{CONCLUSIONES}

En el presente artículo se procuró abordar la concepción toyotista de la producción a partir de la construcción de la productividad que se efectúa en el marco de la relación capital y trabajo y en el seno de las correlaciones de fuerza de la especificidad argentina, a partir del caso de la planta de Toyota localizada en Zárate.

La decisión de problematizar el toyotismo partiendo de cómo se construye la productividad de los sujetos trabajadores pretende matizar ciertos discursos que sostienen la centralidad de las nuevas tecnologías de producción como factor fundamental de la productividad en la fábrica japonesa. Si bien no se niega la incidencia de la microelectrónica y la robotización de ciertos procesos, hacemos hincapié en un enfoque que dé cuenta de que en la construcción de la productividad toyotista, en tanto tecnología de gestión del trabajo, sigue siendo fundamental el uso intensivo de la fuerza de trabajo, mediante la puesta en marcha de sofisticados dispositivos que intervienen sobre los sujetos trabajadores maximizando su capacidad productiva. Remarcando que la disputa por la productividad en el trabajo se juega en gran parte a nivel del sujeto, cabe mencionar algunos hallazgos que sostienen nuestra hipótesis:

- La base objetiva de la intervención sobre el cuerpo de los trabajadores y el tiempo de trabajo mediante dispositivos como la polivalencia, la tercerización y la jornada de trabajo flexible.

- Los aspectos cualitativos que fomentan el involucramiento como variable de productividad mediante la organización del trabajo por células, el kaizen y la formación continua.

- Un sistema complejo de remuneraciones que se calcula a partir de la delimitación de objetivos de productividad y calidad fragmentando la fuerza de trabajo por equipos, y que premia o castiga de manera individualizada el logro o no de los objetivos.

Los mencionados hallazgos sobre estas formas de sujeción en el trabajo confluyen en un articulador común que da sentido a las prácticas de construcción de productividad 
en los sujetos: la flexibilización. En el artículo se dio cuenta de que la flexibilización está presente en los tres niveles mencionados. El uso flexible de los cuerpos y del tiempo de trabajo se pretende legitimar con la ideología de la mejora continua en pos de lograr efectos de consentimiento al sistema de producción. Asimismo, las remuneraciones se flexibilizan y se vuelven dispositivo de individualización de la productividad y la calidad de cada trabajador.

Lo que comanda la dirección flexible de la organización del trabajo son los objetivos de producción que se trazan según la demanda. Es el just in time, el dispositivo técnico-organizacional que marca el ritmo de producción de acuerdo a los vaivenes del mercado. La capacidad de producir por demanda y adaptar a ella la totalidad de los procesos es el factor que explica el alto grado de racionalización del SPT.

El rol que ocupa el sindicato frente a este sistema complejo de racionalización de la fuerza de trabajo es otra de las dimensiones a contemplar en el estudio sobre el toyotismo. La aceptación de la flexibilización juega un papel fundamental para el funcionamiento eficiente del sistema, ya que la existencia de conflictos opera como barrera a su productividad. Las acciones improductivas (el desperdicio) se desbloquean del ámbito específico de la producción y se trasladan al ámbito político-sindical, en el sentido de que la flexibilización es percibida como efecto inamovible de la modernización de las empresas. Es decir, cuestionar la flexibilización desde la política sindical supone cuestionar el eje que estructura el sistema mismo de producción, lo cual, tal como puede observarse en los Convenios Colectivos de Trabajo, resulta inaceptable para la empresa.

Creemos que encarar la cuestión de la flexibilización partiendo del proceso de trabajo y la intervención sobre la subjetividad de los trabajadores nos permite comprender la necesidad que tiene el capital de estructurar el trabajo de manera cada vez más compleja en esta fase del capitalismo global.

\section{REFERENCIAS}

Álvarez, Diego. 2012. "Organización del trabajo y dispositivos de control en el sector automotriz: el toyotismo como sistema complejo de racionalización." Revista Trabajo y Sociedad $18: 43-57$

Alves, Giovanni. 1998. "Reestructuración productiva y crisis del sindicalismo en Brasil." Tesis para optar al grado de Doctor. Campinas: IFCH/UNICAMP. 
Antunes, Ricardo. 2003. ¿Adiós al trabajo? Ensayos sobre las metamorfosis y el rol central del mundo del trabajo. Buenos Aires: Herramienta.

-----. 2005. Los sentidos del trabajo. Buenos Aires: Herramienta.

Battistini, Osvaldo. 2001. “Toyotismo y representación sindical. Dos culturas dentro de la misma contradicción.” Revista venezolana de gerencia 6 (16):553-71.

-----. 2005. "El valor de ser trabajador frente al valor de saber ser." Ponencia presentada en el $7^{\circ}$ Congreso Nacional de Estudios del Trabajo ASET, Buenos Aires.

Coriat, Benjamín. 1992. Pensar al revés. Trabajo y organización en la empresa japonesa. Buenos Aires: Siglo XXI. .

Dirección del Trabajo. 1996a. Convenio Colectivo de Trabajo n 190/96, Art. 16 “Categorías", firmado entre Toyota Argentina Sociedad Anónima y el Sindicato de Mecánicos y Afines del Transporte Automotor.

-----. 1996b. Convenio Colectivo de Trabajo n 190/96, Art. 1.9 “Sistema de Producción Toyota”, firmado entre Toyota Argentina Sociedad Anónima y el Sindicato de Mecánicos y Afines del Transporte Automotor.

-----. 1996c. Convenio Colectivo de trabajo 190/96, 449/2001 y 730/2005 art.1.4 "Responsabilidades de S.M.A.T.A", Firmado entre Toyota Argentina Sociedad Anónima y el Sindicato de Mecánicos y Afines del Transporte Automotor.

-----. 2001a. Convenio Colectivo de Trabajo $n^{\circ}$ 449/2001, Art. 17 "Funciones y Clasificaciones", firmado entre Toyota Argentina Sociedad Anónima y el Sindicato de Mecánicos y Afines del Transporte Automotor.

-----. 2001b. Convenio Colectivo de Trabajo $n^{\circ} 449 / 2001$, Art. 19 "Polivalencia funcional y multioficios", firmado entre Toyota Argentina Sociedad Anónima y el Sindicato de Mecánicos y Afines del Transporte Automotor.

----. 2005a. Convenio Colectivo de Trabajo n 730/2005, Anexo "B” al Art. 35 "Criterios para el traspaso de niveles - Factores considerados al momento de la evaluación”, firmado entre Toyota Argentina Sociedad Anónima y el Sindicato de Mecánicos y Afines del Transporte Automotor.

----. 2005b. Convenio Colectivo de Trabajo n 730/2005, Anexo “B” al Art. 35 "Criterios para el traspaso de niveles - Factores considerados al momento de la evaluación”, firmado 
entre Toyota Argentina Sociedad Anónima y el Sindicato de Mecánicos y Afines del Transporte Automotor.

-----. 2005c. Convenio Colectivo de Trabajo n 730/2005, Art 31.1 “Cumplimiento de la jornada de trabajo”, firmado entre Toyota Argentina Sociedad Anónima y el Sindicato de Mecánicos y Afines del Transporte Automotor.

2005d. Convenio Colectivo de Trabajo $n^{\circ} 730 / 2005$, Art 31.2 “Jornada específica de trabajo", firmado entre Toyota Argentina Sociedad Anónima y el Sindicato de Mecánicos y Afines del Transporte Automotor.

-----. 2005e. Convenio Colectivo de Trabajo $n^{\circ} 730 / 2005$, Art 33 “Modalidad programada de trabajo", firmado entre Toyota Argentina Sociedad Anónima y el Sindicato de Mecánicos y Afines del Transporte Automotor.

-----. 2005f. Convenio Colectivo de Trabajo $n^{\circ}$ 730/2005, Art 1.10 "Kaizen", firmado entre Toyota Argentina Sociedad Anónima y el Sindicato de Mecánicos y Afines del Transporte Automotor.

-----. 2005g. Convenio Colectivo de Trabajo n 730/2005, Art 35.2 b “Sistema de pagos por productividad y calidad. Remuneración variable", firmado entre Toyota Argentina Sociedad Anónima y el Sindicato de Mecánicos y Afines del Transporte Automotor.

-----.2005h. Convenio Colectivo de Trabajo n 730/2005, Anexo A del Art 35.2 b “Sistema de pagos por productividad y calidad. Remuneración variable”, firmado entre Toyota Argentina Sociedad Anónima y el Sindicato de Mecánicos y Afines del Transporte Automotor.

-----2005i. Convenio Colectivo de Trabajo $n^{\circ}$ 730/2005, Anexo B del Art 35.2 a.2 "Criterios para el traspaso de niveles", firmado entre Toyota Argentina Sociedad Anónima y el Sindicato de Mecánicos y Afines del Transporte Automotor.

Escobar, Areli. 2004. "La subjetividad de los trabajadores en la era del toyotismo." Revista de la Academia 9:67-76.

Figari, Claudia. 2007. "Procesos de racionalización, disciplinamiento laboral y dispositivos de control social, entre la individualización y la normalización del trabajo." Ponencia presentada en el V Congreso Latinoamericano de Sociología del Trabajo, Buenos Aires.

Figari, Claudia y Marcelo Hernández. 2008. "Dispositivos de disciplinamiento laboral en una empresa del sector automotriz. El "mantenimiento total” como espacio de disputa." Ponencia presentada en el $4^{\circ}$ Seminario de Trabajo UNESP-MARILLA, Sao Paulo. 
Foucault, Michel. 2002. Vigilar y castigar, nacimiento de la prisión. Buenos Aires: Siglo XXI.

Gorz, André. 1998. Miserias del presente, riquezas de lo posible. Buenos Aires: Paidós.

Ichiyo, Muto. 1997. "Luchas de clase en el Japón de posguerra. Pasado, presente y futuro." En Japón: ¿milagro o pesadilla? La otra cara del toyotismo, compilado por O. Martínez. Buenos Aires: Taller de Estudios Laborales.

Juárez, Humberto. 2002. “Los sistemas Just in time/Kanban, un paradigma productivo.” Política y Cultura 18:40-60.

Lipietz, Alain. 1994. "El posfordismo y sus espacios. Las relaciones capital-trabajo en el mundo.” Documento de Trabajo N 4 . Buenos Aires: PIETTE-CONICET.

Martínez, Oscar. 1997. Japón ¿milagro o pesadilla? La otra cara del toyotismo. Buenos Aires: Taller de Estudios Laborales.

Novick, Marta, Gabriel Yoguel, Ana Catalano y Facundo Albornoz. 2004. "Adaptación de modelos productivos en países emergentes. El caso de la industria automotriz en la Argentina." Sociologie du travail 46.

Totsuka, Hideo. 1997. "La sociedad corporativa japonesa." En Japón: ¿milagro o pesadilla? La otra cara del toyotismo, compilado por O. Martínez. Buenos Aires: Taller de Estudios Laborales.

Watanabe, Ben. 1997. “Organizar lo desorganizado.” En Japón: ¿milagro o pesadilla? La otra cara del toyotismo, compilado por O. Martínez. Buenos Aires: Taller de Estudios Laborales.

Wortman, Ana. 2003. "Subjetividad y sociedad de consumo en la Argentina de los 90. El impacto del toyotismo." e-latina, Revista Electrónica de Estudios Latinoamericanos número:1-2. 